\title{
$\omega-3$ E $\omega-9$ SOBRE O DESEMPENHO, RESPOSTA SUPEROVULATÓRIA E PRODUÇÃO DE EMBRIÕES EM NELORE
}

\author{
$\omega-3$ AND $\omega-9$ ON PERFORMANCE, SUPEROVULATORY RESPONSE AND EMBRYO \\ PRODUCTION IN NELLORE HEIFERS
}

\author{
Muller, M. ${ }^{1}$, I.N. Prado ${ }^{2 A}$, A.R. Lobo Júnior ${ }^{2}$, R.R. Silva ${ }^{4 A}$, L.C.T. Capovilla ${ }^{3}$, L.P. Rigolon ${ }^{3}$, \\ F.L.B. Cavalieri ${ }^{3}$ e J.A. Marques ${ }^{4}$
}

\begin{abstract}
${ }^{1}$ Pontifícia Universidade Católica do Paraná (PUCPR). Campus Toledo. Av. da União, 500. CEP 85902-532. Toledo/PR. Brasil. mylene@rla01.pucpr.br

${ }^{2}$ Universidade Estadual de Maringá. Av. Colombo, 5790. Jd. Universitário. CEP 87020-900. Maringá. Paraná. Brasil. Ainprado@uem.br

${ }^{3}$ CESUMAR. Av. Guedner, 1610. Jardim Aclimação. CEP 87050-390. Maringá. Paraná. Brasil. fbin@cesumar.br

${ }^{4}$ Universidade Estadual do Sudoeste da Bahia. Pça Primavera, 40. Bairro Privamera. CEP 45700-000. Itapetinga. Bahia. Brasil. Arrsilva.uesb@hotmail.com
\end{abstract}

\section{PalaVRas chaVe ADICIONAIS}

Semente de linhaça. Semente de canola.

\section{RESUMO}

Foram utilizadas 17 novilhas Nelore, com 20 meses de idade e peso médio inicial de $343,9 \mathrm{~kg}$, durante 28 dias, para estudar o efeito de fontes de gordura ômega-3 (semente de linho, LIN), ômega9 (semente da canola, CAN) e sem adição de gordura (SGO) sobre o peso vivo final, ganho médio diário, espessura de gordura de corbertura, número e peso de corpos lúteos e cistos ováricos, número de estruturas totais, transferíveis, degeneradas e não fertilizadas. As novilhas foram distribuídas em delineamento inteiramente casualizado. Não houve efeito das fontes LIN e CAN e SGO sobre o peso vivo final, ganho médio diário e espessura de gordura. Não houve diferença sobre os pesos do ovário, corpo lúteo e cisto, esquerdo, direito e total e para o número de corpo lúteo total e cistos no ovário esquerdo. O CAN apresentou maior número de corpo lúteo esquerdo $(18,33$ vs. 12,00) e menor número de cistos no ovário direito $(1,33$ vs. 4,60$)$ e total $(3,60$ vs. 8,60$)$ comparado ao LIN. As novilhas do CAN e SGO apresentaram maior número de estruturas totais $(8,67$ e 10,40). O CAN apresentou maior número de estruturas transferíveis $(6,67)$ em relação ao SGO e LIN $(2,80$ vs. 1,17$)$. LIN e CAN apresentaram

Recibido: 30-7-07. Aceptado: 1-4-08.

\section{AdDitional KEYWORDS}

Linseed. Canola seed.

menor número de estruturas degeneradas em relação ao SOG (2,00 e 3,67 vs. 7,60). No entanto, o CAN apresentou menor número de estruturas não fecundadas $(0 \%)$ e maior viabilidade das estruturas $(76,93 \%)$.

\section{SUMMARY}

Twenty three 20 months old and $351 \mathrm{~kg}$ of initial body weight Nellore heifers were used, during 28 days, to study the effect of omega-3 (whole linseed, LIN), omega-9 (canola seed, CAN) fat sources, and without fat addition (no fat, SOG) on the final live weight, average daily gain, fat tickness, number and weight of luteum corpus and cyst, number of total structures, transferable structures, degenerate structures, and no-fertilized structure. The heifers were allotted into a completely randomized design. There was no effect of LIN and CAN fat sources and SOG on the final body weight, average daily gain, and fat thickness. There was no difference on weight of left, right, and total ovarium, corpus luteum and cyst; and on number of total luteum corpus and left ovarium cyst. CAN presented a higher number of left luteum 


\section{MULLER ET AL.}

corpus (18.33 vs. 12.00$)$ and a lower number of right ovarium cyst (1.33 vs. 4.60$)$ and total (3.60 vs. 8.60), compared to LIN. CAN e SOG had a higher number of total structure (8.67 and 10.40). CAN had a higher number of transferable structures (6.67) than SOG and LIN (2.80 vs. 1.17). LIN and CAN had a lower number of degenerate structure than SOG (2.00 and 3.67 vs. 7.60). Therefore, CAN presented a lower number of non-fertilized structure $(0 \%)$ and a higher viability of produced structure $(76.93 \%)$.

\section{INTRODUÇÃO}

A adição de gordura na dieta aumenta a ingestão de energia líquida e, portanto, decresce a duração e a magnitude do status energético negativo (Oldick et al., 1997), melhorando o desempenho animal (Zinn e Plascencia, 1996). Ainda, a inclusão de gordura na dieta, pode alterar o desempenho reprodutivo de ruminantes. Estes efeitos podem ser dependentes do perfil de ácidos graxos da fonte de gordura (Oldick et al., 1997) e, também, da relação de causa e efeito entre a progesterona e o colesterol. A dieta alta em gordura aumenta as concentrações de colesterol sangüíneo e, este é um dos precursores de progesterona (Lammoglia et al., 2000).

A suplementação de gordura tem sido estudada por causar efeitos benéficos na reprodução, como o aumento na concentração sangüínea de progesterona, tamanho do folículo ovulatório, número de folículos ovarianos, modulação do corpo lúteo, taxa de concepção e gestação (Staples et al., 1996). Estes efeitos positivos foram observados por Thomas et al. (1997) ao testarem três fontes de gordura: gordura saturada, óleo de soja e óleo de peixe, contra o tratamento testemunha (sem inclusão de gordura), em vacas recebendo dietas isoenergéticas e isoprotéicas. Os autores verificaram que a inclusão de $4 \%$, na base da matéria seca, de óleo de soja aumentou quatro vezes o número de folículos médios (4,0 a 9,9 mm de diâmetro) em relação ao grupo controle.
Estudos realizados por Thomas e Williams (1996), ao testarem o efeito de três dietas (controle, óleo de soja e gordura animal) na resposta superovulatória de 21 novilhas, sendo o folículo dominante puncionado para diminuir o seu efeito, mostraram que o fornecimento de gordura, tanto de origem vegetal quanto animal, aumentou o número de folículos médios. Para os animais que receberam óleo de soja houve um aumento na concentração de insulina no sangue, mas este não alterou o número de embriões coletados.

Pesquisas realizadas por Petit et al.(1998) mostraram que o perfil de ácidos graxos altera a taxa de prenhez. Os autores encontraram taxas de prenhez de $50 \%$, quando houve suplementação de fonte de ácidos graxos ômega-6 (ácido linoléico), e de $89 \%$ quando a fonte foi de ômega-3 (ácido linolênico). Estes mecanismos podem ser mediados pela redução na síntese de prostaglandina, pois esta é sintetizada a partir dos ácidos graxos, embora os mecanismos exatos envolvidos ainda sejam desconhecidos (Petit et al., 2002).

O objetivo deste trabalho foi avaliar o efeito das fontes de gordura ômega-3 (semente de linho) e ômega-9 (semente de canola) sobre o desempenho e produção de embriões em novilhas da raça Nelore.

\section{MATERIAL E MÉTODOS}

Local: o experimento foi realizado no setor de Bovinocultura de Corte da Fazenda da Experimental de Iguatemi (FEI) da Universidade Estadual de Maringá (UEM), localizada no distrito de Iguatemi, Maringá, no Estado do Paraná. As análises bromatológicas dos alimentos foram realizadas no Laboratório de Nutrição Animal da UEM e as análises dos ácidos graxos foram realizadas no Laboratório de Análise de Alimentos do Departamento de Química da UEM.

Animais: foram utilizadas 17 novilhas Nelore com idade média de 20 meses e peso vivo médio de $343,90 \mathrm{~kg}$. Antes do início do 
FONTES DE GORDURA SOBRE DESEMPENHO E PRODUÇÃO DE EMBRIÕES

Tabela I. Composição química (\% MS) dos alimentos e das rações. (Chemical composition (\%/ DM) of the ingredients and diets).

\begin{tabular}{|c|c|c|c|c|c|c|c|c|}
\hline Ingredientes & MS & PB & EE & FDN & FDA & HEM & MO & MM \\
\hline Silagem de milho & 32,2 & 7,8 & 2,7 & 53,9 & 30,0 & 23,9 & 95,1 & 5,0 \\
\hline Milho & 84,9 & 10,5 & 1,0 & 12,0 & 3,9 & 8,1 & 99,0 & 1,0 \\
\hline Farelo de soja & 89,7 & 49,5 & 1,6 & 12,9 & 8,3 & 4,5 & 96,0 & 4,0 \\
\hline Resíduo de fecularia & 88,9 & 2,1 & 0,1 & 32,8 & 22,5 & 10,2 & 98,5 & 1,5 \\
\hline Semente de linho & 93,1 & 28,9 & 41,0 & 15,1 & 11,7 & 3,4 & 96,2 & 3,8 \\
\hline Semente de canola & 92,7 & 24,5 & 35,5 & 17,8 & 12,7 & 5,2 & 95,7 & 4,4 \\
\hline Uréia & 98,0 & 262,0 & - & - & - & - & - & - \\
\hline \multicolumn{9}{|l|}{ Rações } \\
\hline $\mathrm{SGO}^{1}$ & 43,1 & 10,8 & 1,9 & 41,2 & 23,5 & 17,7 & 95,2 & 4,8 \\
\hline $\operatorname{Lin}^{2}$ & 42,0 & 12,0 & 5,8 & 41,3 & 23,6 & 17,8 & 94,7 & 5,3 \\
\hline $\mathrm{Can}^{3}$ & 42,1 & 11,6 & 5,2 & 41,4 & 23,5 & 17,9 & 94,6 & 5,4 \\
\hline
\end{tabular}

${ }^{1}$ Sem adição de gordura; ${ }^{2}$ Semente de linho; ${ }^{3}$ Semente da canola.

experimento, as novilhas foram pesadas, identificadas com brincos plásticos na orelha esquerda e tratadas com Ivermectina $1 \%$ $\left(\right.$ Ivomec, Merial ${ }^{\circledR}$ ). As novilhas foram alojadas em baias individuais de $10 \mathrm{~m}^{2}$, com piso de concreto, cercadas com barras de ferro, com área coberta com telhas de zinco e solário.

Alimentação: a alimentação foi fornecida em comedouros de alvenaria com 2 m linear/ animal. A água foi fornecida ad libitum em bebedouros localizados na área descoberta das instalações. Sessenta dias antes de iniciar o tratamento superovulatório, as novilhas foram distribuídas, aleatoriamente, para receber um dos tratamentos: SGO: sem adição de gordura $(n=6)$, LIN: semente de linho $(n=6)$ e CAN: semente de canola $(n=5)$. As dietas foram formuladas de acordo com as recomendações do NRC (1996), mantendo-as com quantidades próximas de energia e proteína. A ração foi fornecida às $8 \mathrm{~h}$ e $16 \mathrm{~h}$. No período da manhã foram retiradas as sobras para determinação do consumo de matéria seca. A ração começou a ser fornecida 60 dias antes de iniciar o tratamento superovulatório, tempo suficiente para ocorrer à adaptação dos animais às dietas. A alimentação do tratamento SGO era constituída de grão de milho, farelo de soja, resíduo de fecularia de mandioca, uréia, calcário e sal mineral 90. O tratamento LIN foi constituído de semente de linho (fonte de ômega-3), grão de milho, farelo de soja, resíduo de fecularia de mandioca, uréia, calcário e sal mineral 90; e o tratamento CAN de semente de canola (fonte de ômega-9), grão de milho, farelo de soja, resíduo de fecularia de mandioca, uréia, calcário e sal

Tabela II. Composição (\%na MS) das rações experimentais. (Composition (\%/DM) of the experimental diets).

\begin{tabular}{lccc}
\hline Ingredientes & SOG $^{1}$ & LIN $^{2}$ & CAN $^{3}$ \\
\hline Silagem de milho (MS) & 60,2 & 63,7 & 63,2 \\
Milho & 12,2 & 10,8 & 11,5 \\
Farelo soja & 6,9 & 2,7 & 2,4 \\
Resíduo de fecularia & 19,6 & 12,0 & 12,1 \\
Semente de linho & - & 9,4 & - \\
Semente de canola & - & - & 9,3 \\
Uréia & 0,4 & 0,6 & 0,7 \\
Calcário & 0,4 & 0,4 & 0,4 \\
Sal mineral & 0,4 & 0,4 & 0,4 \\
\hline
\end{tabular}

${ }^{1}$ Sem adição de gordura; ${ }^{2}$ Semente de linho; ${ }^{3}$ Semente da canola. 


\section{MULLER ET AL.}

mineral 90. A composição química dos alimentos e das rações encontra-se na tabela I e a composição percentual das rações na tabela II.

Programa de superovulação: após 60 dias de experimento, os animais foram submetidos à superovulação, iniciando com implante auricular de 3,0 mg de Norgestomet $\left(\right.$ Crestar $^{\circledR}$, Intervet SA). No dia seguinte, foi administrada, via intramuscular, 2,5 $\mathrm{mg}$ de valerato de estradiol. Cinco dias após a administração do estradiol, as novilhas foram submetidas ao tratamento superovulatório com 250 UI total de hormônio folículo estimulante (FSH), em duas doses diárias, sendo uma a cada 12 horas (tabela III). A dose total de FSH (250 UI) foi dividida em quatro dias, com doses decrescentes, sendo que na quinta aplicação foi retirados o implante e aplicado $2 \mathrm{ml} \mathrm{de} \mathrm{PGF}_{2 \alpha}\left(\right.$ Veteglan $\left.^{\circledR}\right)$, via intramuscular.

As novilhas foram observadas às $8 \mathrm{~h}, 14$ $\mathrm{h}$ e $17 \mathrm{~h}$, com o objetivo de detectar qualquer sinal de estro. Após, as fêmeas foram inseminadas 12 e 24 horas após a identificação do estro, com sêmen fresco de um único touro, da raça Holandês, sendo o mesmo previamente analisado, com qualidade comprovada ( $70 \%$ de motilidade progressiva e vigor de 4 pontos).

Coleta de embrióes: antes da coleta dos embriões as novilhas foram submetidas à palpação retal para avaliar a resposta superovulatória mediante ao número de corpos lúteos encontrados. A coleta dos embriões foi realizada pelo método não cirúrgico, no sétimo dia após a inseminação artificial. A lavagem uterina foi realizada com $500 \mathrm{ml}$ do meio Dulbecco modificado (PBS), aquecido a $37^{\circ} \mathrm{C}$ e enriquecido com $0,2 \%$ de BSA. O mesmo foi introduzido com o auxílio de um cateter de Folley de duas entradas, número 18 , através da cérvice. $\mathrm{O}$ efluente recolhido foi filtrado em filtro de 75 $\mathrm{m}$ e os embriões foram colocados dentro de uma placa de Petri quadriculada. Estes foram observados em microscópio estereoscópio em 40 aumentos para a localização e de 90 vezes para a avaliação das estruturas embrionárias quanto ao estádio de desenvolvimento e a qualidade, segundo a classificação proposta pelo manual do IETS, International Embryo Transfer Society (1999): grau 1 (excelente ou bom), grau 2

Tabela III. Protocolo do tratamento superovulatório. (Superovulatory treatment protocol).

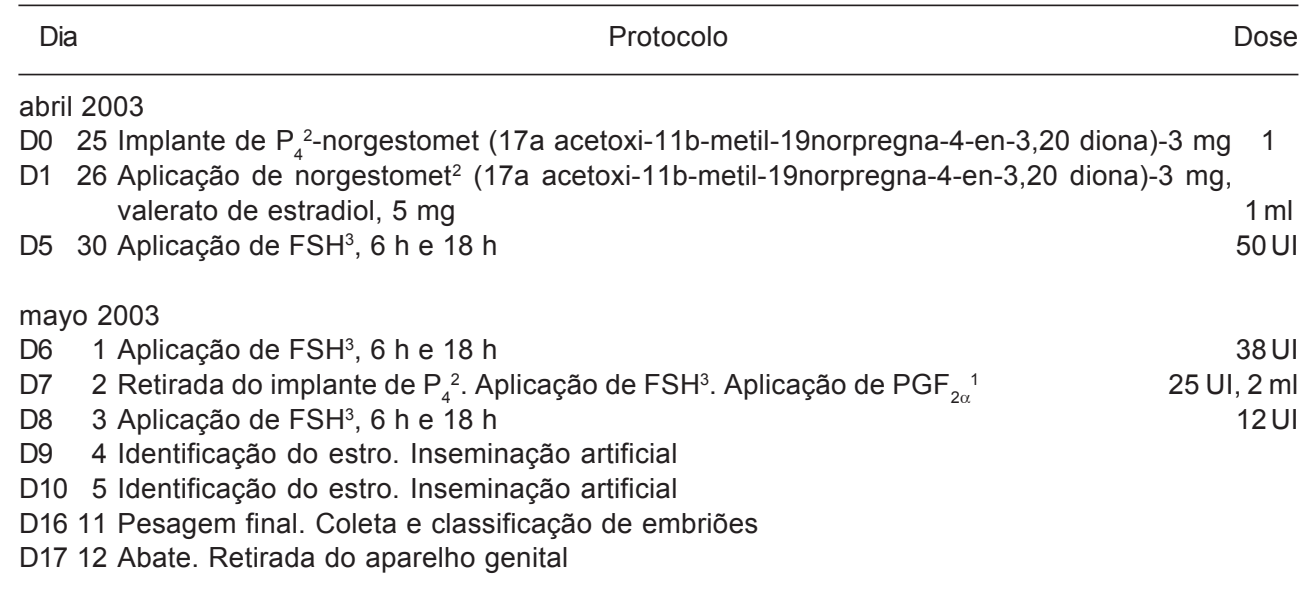

${ }^{1}$ Veteglan ${ }^{\circledR} ;{ }^{2}$ Crestar $^{\circledast} ;{ }^{3}$ Pluset ${ }^{\circledR}$.

Archivos de zootecnia vol. 58, núm. 222, p. 244. 
(regular), grau 3 (pobre) e grau 4 (infertilizados).

No dia seguinte, a coleta dos embriões, as novilhas foram submetidas a um jejum de sólidos e líquidos de 10 horas antes do embarque para o frigorífico, para a realização do abate. Após o abate, foram coletados e identificados os tratos reprodutivos dos animais e levados imediatamente ao Laboratório de Reprodução Animal da UEM, para a realização da contagem e pesagem dos corpos lúteos, cistos e ovários (esquerdo e direito).

Vinte e quato horas após o abate e resfriamento a $-4^{\circ} \mathrm{C}$, foi mensurado, a espessura de gordura de cobertura (EGC) das amostras do músculo longissimus dorsi entre a $12^{\mathrm{a}}$ e $13^{\mathrm{a}}$ costelas (Müller, 1987), para avaliação da deposição de gordura dos animais.

Análises estatísticas: $\mathrm{O}$ delineamento experimental foi inteiramente casualizado. A análise dos dados foi realizada utilizando o programa estatístico Statistical Analysis System (SAS Institute, 1996). Os pesos e ganhos de peso das novilhas, os pesos dos ovários, corpos lúteos e cistos, foram analisados pelo método dos quadrados mínimos, utilizando o procedimento General Linear Models (GLM), que considera o desbalanceamento do número de observações (SAS Institute, 1996), sendo o peso vivo inicial usado como covariável. O modelo estatístico utilizado foi o seguinte:

$$
Y_{i j}=\mu+T_{i}+e_{i i k}
$$

onde:

$\mu=$ constante geral associada a cada observação; $Y_{i j}=$ observação referente ao animal j, submetido ao tratamento $i$ ( $i=1,2$ e 3 );

$T_{i}=$ efeito do tratamento $i(i=1,2$ e 3$)$;

$E_{i j k}=$ erro aleatório associado a cada observação.

O número de corpos lúteos, cistos, estruturas totais, estruturas transferíveis, estruturas degeneradas e não fecundadas, foram analisadas pelo teste do $\chi^{2}$ (Gomez e Gomez, 1984), utilizando o procedimento GENMOD do SAS.

\section{RESULTADOSEDISCUSSÃO}

O peso vivo final, o ganho médio diário e a espessura de gordura de cobertura das novilhas não foram influenciados $(\mathrm{p}>0,05)$ pelos tratamentos (tabela IV).

No entanto, deve ser salientando que as diferentes rações eram isoprotéicas e isoenergéticas, ocorrendo diferenças apenas nas fontes de energia (milho ou semente de linho ou semente de canola). Portanto, os ganhos obtidos para os tratamentos sem gordura $(1,29 \mathrm{~kg})$, semente de linho $(1,24 \mathrm{~kg})$ e semente de canola $(1,13 \mathrm{~kg})$ estão de acordo

Tabela IV. Fontes de gordura sobre o peso vivo inicial (PVI), peso vivo final (PVF), ganho médio diário (GMD) e espessura de gordura de cobertura (EGC) das novilhas. (Fat sources on initial body weight (IBW), final body weight (FBW), average daily gain (ADG) and fat thickness (FT) of heifers).

\begin{tabular}{lccccc}
\hline Variáveis* & SOG $^{*}$ & LIN $^{2}$ & CAN $^{3}$ & Média & CV $(\%)^{* *}$ \\
\hline N $^{*}$ novilhas & 6 & 6 & 5 & - & - \\
PVI $(\mathrm{kg})$ & 343,3 & 351,6 & 337,0 & 357,3 & 11,4 \\
PVF $(\mathrm{kg})$ & 388,9 & 387,4 & 383,9 & 386,7 & 15,8 \\
GMD $(\mathrm{kg})$ & 1,3 & 1,2 & 1,1 & 1,2 & 15,7 \\
EGC $(\mathrm{mm})$ & 9,1 & 7,1 & 8,3 & 8,2 & 29,7
\end{tabular}

${ }^{1}$ Sem gordura; ${ }^{2}$ Semente de linho; ${ }^{3}$ Semente de canola; ${ }^{*}$ Não houve diferença significativa. ${ }^{* *}$ Coeficiente de variação. 


\section{MULLER ET AL.}

com os resultados esperados.

Zinn e Plascencia (1996), ao testarem dois níveis de inclusão de gordura amarela $(0 \%$ ou $6 \%)$ e dois níveis de feno de alfafa (10 e 30\%) em 96 novilhos cruzados, com peso vivo médio de $316 \mathrm{~kg}$, constataram que a adição de gordura na dieta alta em feno aumentou o ganho médio diário em 13,3\%. Da mesma forma, Zinn (1989) constatou que a inclusão de diferentes fontes de gordura (4\% de gordura amarela, $4 \%$ de uma mistura de gordura animal + vegetal, $8 \%$ de gordura amarela, $8 \%$ de mistura de gordura vegetal + animal e $6 \%$ de mistura de gordura vegetal + animal $+2 \%$ de milho + lecitina de soja) aumentou linearmente o ganho de peso de novilhos mestiços, com peso vivo médio de $333 \mathrm{~kg}$, terminados durante 125 dias, em confinamento. Por outro lado, Petit et al. (1997) não observaram efeito da adição de semente de canola ou grão de soja sobre o peso vivo e ganho médio diário em cordeiros inteiros terminados em confinamento.

Embora, nos últimos 10 dias de confinamento o manejo das novilhas tenha sido intenso, em função do tratamento superovulatório (tabela III), o ganho médio diário obtido pelas mesmas independentemente do tratamento foi elevado $(1,22 \mathrm{~kg})$. Deve-se ainda salientar que, em função do tratamento superovulatório, todas as novilhas manifestaram cio o que poderia resultar no menor ganho em peso, como observado por Prado et al. (2000) em novilhas confinadas e alimentadas ad libitum. O ganho observado neste experimento foi superior ao ganho observado por Medroni et al. (2000), utilizando milho, como fonte de energia e farelo de soja como fonte de proteína $(1,14 \mathrm{~kg})$, em novilhas mestiças terminadas em confinamento. Prado et al. (2000) e Marques et al. (2001), trabalhando com novilhas da mesma categoria e peso semelhante, terminadas em confinamento e alimentadas com diferentes fontes de energia (milho ou subprodutos da mandioca) e proteína (farelo de soja ou levedura de cana-de-açúcar) observaram ganhos em peso inferiores ao deste experimento.

As novilhas apresentavam elevado score corporal proporcionado uma espessura de gordura de cobertura de $8,20 \mathrm{~mm}$ (tabela IV), sem diferenças entre tratamentos $(\mathrm{p}>0,05)$.

O número de novilhas que responderam

Tabela $V$. Fontes de gordura sobre peso do ovário direito, esquerdo e total, peso de corpo lúteo dos ovários direito, esquerdo e total e peso de cistos dos ovários direito, esquerdo e total das novilhas. (Fat sources on right, left and total ovarium weight, right, left and total corpus luteum and right, left ant total cyst of heifers).

\begin{tabular}{lccccc}
\hline Item & SOG & LIN $^{*}$ & CAN $^{3}$ & Média & CV $(\%)^{* *}$ \\
\hline N $^{\circ}$ novilhas & 6 & 6 & 5 & - & - \\
N $^{\circ}$ novilhas CL & 5 & 6 & 3 & - & - \\
Ovário direito (g) & 27,4 & 33,0 & 27,6 & 29,3 & 64,4 \\
Ovário esquerdo (g) & 31,8 & 30,0 & 43,1 & 35,0 & 57,6 \\
Ovário total (g) & 59,2 & 63,0 & 70,7 & 64,3 & 58,0 \\
Corpo lúteo direito (g) & 14,7 & 18,2 & 18,7 & 17,2 & 69,1 \\
Corpo lúteo esquerdo (g) & 19,7 & 15,3 & 26,7 & 20,6 & 60,1 \\
Corpo lúteo total (g) & 34,4 & 33,5 & 45,4 & 3,8 & 61,3 \\
Cisto ovário direito (g) & 2,4 & 8,0 & 1,8 & 4,1 & 103,0 \\
Cisto ovário esquerdo (g) & 3,5 & 8,1 & 6,2 & 5,9 & 89,8 \\
Cisto ovário total (g) & 5,8 & 16,0 & 8,0 & 9,9 & 82,3 \\
\hline
\end{tabular}

${ }^{1}$ Sem de gordura; ${ }^{2}$ Semente de linho; ${ }^{3}$ Semente de canola; *Não houve diferença significativa. ${ }^{* *}$ Coeficiente de variação.

Archivos de zootecnia vol. 58, núm. 222, p. 246. 
ao tratamento superovulatório foi de $5 / 6$ $(83,3 \%)$ no tratamento sem gordura, $6 / 6$ $(100,0 \%)$ no tratamento com semente de linhaça e $3 / 5(60,0 \%)$ no tratamento com semente de canola (tabela V).

Os dados demonstram que a inclusão de semente de linhaça na dieta de novilhas, durante 84 dias, proporcionou um maior número de novilhas com corpo lúteo. Por outro lado, para as novilhas suplementadas com semente de canola o efeito responsivo foi negativo. A semente de canola (Brassica napus L.) é uma variedade geneticamente modificada da semente de colza, possuindo desta forma quantidade inferior a $2 \%$ de ácido erúcico (total dos ácidos graxos) e, menos que $3 \mathrm{mg} / \mathrm{g}$ de matéria seca em glicosinolatos (Bett et al., 1999). Todavia, por se tratar de novilhas, iniciando a maturação do ambiente uterino (Staigmiller et al., 1993), as concentrações de ácido erúcico e glicosinolatos podem ter prejudicado o sincronismo de comunicação entre útero e ovário (Del Vecchio et al., 1990), mesmo em pequenas concentrações. Ainda, em função da semente de canola conter somente $8,6 \%$ de ácido linolênico e 20\% de ácido linoléico (Downey, 1990), pode ter ocorrido uma secreção prematura de prostaglandina (Petit et al., 2002), pois esta exerce o papel de regressão funcional do corpo lúteo (Oldick et al., 1997). No entanto, os mecanismos envolvidos na síntese de prostaglandina ainda são complexos (Petit etal., 2002).

Ao contrário da semente de canola, a linhaça possui $52 \%$ do total dos ácidos graxos na forma de ácido linolênico (Romans et al., 1995), o que segundo Petit et al. (2002), resulta em maior número de folículos entre 5,0 e 9,9 mm de diâmetro e aumento no tamanho do maior folículo quando esta foi associada ao óleo de peixe (50:50).

Não foi observado $(p>0,05)$ efeito de tratamento sobre o peso do ovário direito, esquerdo e total. O peso médio do ovário (direito + esquerdo), das três dietas, foi de 63,34 g. Este peso elevado dos ovários mostra que houve uma boa resposta superovulatória em resposta conseqüência do estímulo ao tratamento hormonal. Em bovinos não superovulados, Ohashi et al. (1998), encontraram valores de 5,4 g de peso total de ovários.

$O$ peso da massa de corpo lúteo do ovário direito foi semelhante $(\mathrm{p}>0,05)$ para as novilhas dos três tratamentos (tabela $\mathbf{V}$ ). Por outro lado, o peso da massa de corpo lúteo do ovário esquerdo, embora não tenha revelado diferença significativa, em função da alta variabilidade dos dados (coeficiente de variação $=60 \%$ ) para novilhas suplementadas com semente de canola foi $58 \%$ superior ao peso da massa de corpo lúteo das novilhas do demais tratamentos. Em função destes dados, comportamento semelhante foi observado para o peso da massa total de corpo lúteo $(45,44 \mathrm{~g}$ para o tratamento com semente de canola vs. $34,42 \mathrm{~g}$ para o tratamento sem gordura e 33,49 g para o tratamento com semente de linhaça.

Petit et al. (2002), utilizando quatro vacas da raça Holandesa, canuladas no rúmen e duodeno, recebendo $60 \%$ de silagem de azevém e $40 \%$ de concentrado para testar o efeito de quatro tipos de concentrados: Megalac $\AA$, semente de linho tratada com formaldeído, uma mistura de $50 \%$ de óleo de peixe e $50 \%$ de semente de linho tratada com formaldeído e concentrado sem adição de gordura, com infusão duodenal de $500 \mathrm{~g}$ de óleo de linho/dia, constataram que o tamanho médio do corpo lúteo foi maior para as vacas que receberam maiores quantidades de ômega-3 na dieta (semente de linho) divergindo dos dados encontrados neste experimento. Entretanto, não ficou claro para os autores por que das vacas infundidas com óleo de linho apresentarem um tamanho de corpo lúteo menor que as vacas alimentadas com semente de linho.

$\mathrm{O}$ peso da massa de cistos do ovário direito, esquerdo e total foi maior $(p<0,05)$ para as novilhas do tratamento com semente de linhaça ( $175 \%$ em relação ao tratamento sem gordura e $100 \%$ em relação ao trata- 


\section{MULLER ET AL.}

mento com semente de canola, tabela $\mathbf{V}$ ). Provavelmente, a maior massa de folículos não ovulatórios contribuiu para o menor número de estruturas totais no LIN (tabela VII).

O número de corpo lúteo encontrado no ovário direito das novilhas foi semelhante ( $p>0,05)$ entre os tratamentos sem gordura, com semente de linhaça e semente de canola (tabela VI). No entanto, no ovário esquerdo o número de corpo lúteo encontrado foi maior $(\mathrm{p}<0,05)$ para as novilhas suplementadas com canola $(18,3)$ em relação às novilhas suplementadas com semente de linho (12,0). Ainda, não houve diferença $(p>0,05)$ entres as novilhas sem gordura e àquelas suplementadas com semente de linho ou semente de canola. Embora o número de corpo lúteo tenha sido maior no ovário esquerdo para as novilhas suplementadas com semente de canola, o número total de corpo lúteo (ovário direito + ovário esquerdo) foi semelhante para os três tratamentos (tabela VI).

Tabela VI. Fontes de gordura sobre o número de corpo lúteo no ovário direito, esquerdo e total, número de cistos no ovário direito, esquerdo e total. (Fat sources on number of right, left and total corpus luteum, number of right, left and total cyst).

\begin{tabular}{lccc}
\hline Item & SGO & LIN & CAN \\
\hline$N^{\circ}$ novilhas & 6 & 6 & 5 \\
$\mathrm{~N}^{\circ}$ novilhas CL & 5 & 6 & 3 \\
$\quad \mathrm{~N}^{\circ}$ corpo lúteo ovário & & & \\
$\quad$ direito & 13,4 & 14,6 & 14,0 \\
$\quad$ esquerdo & $14,6^{\mathrm{ab}}$ & $12,0^{\mathrm{b}}$ & $18,3^{\mathrm{a}}$ \\
$\quad$ total & 28,0 & 26,6 & 32,3 \\
$\mathrm{~N}$ ㅇstos ovário & & & \\
$\quad$ direito & $1,4^{\mathrm{a}}$ & $4,6^{\mathrm{b}}$ & $1,3^{\mathrm{a}}$ \\
$\quad$ esquerdo & 2,8 & 4,0 & 2,3 \\
$\quad$ totais & $4,2^{\mathrm{a}}$ & $8,6^{\mathrm{b}}$ & $3,7^{\mathrm{a}}$ \\
\hline
\end{tabular}

${ }^{1}$ Sem de gordura; ${ }^{2}$ Semente de linho; ${ }^{3}$ Semente de canola.

ab $\mathrm{Na}$ mesma linea, médias com letras diferentes apresentaram diferença estatística $(p<0,05)$.
Lammoglia et al. (1997), testando uma dieta controle ( $3,7 \%$ EE) e uma dieta suplementada com farelo de arroz $(5,2 \% \mathrm{EE}) \mathrm{em}$ vacas Brahman, verificaram que os animais que receberam a fonte de gordura aumentaram a população folicular na primeira onda do segundo ciclo estral e as concentrações de insulina e colesterol. Todavia, Lammoglia et al. (2000), suplementando 246 novilhas, de três grupos genéticos diferentes (Hereford, Limousin e Piedmontese), com uma dieta baixa $(1,9 \%$ EE) e uma alta $(4,4 \%$ EE) de gordura, utilizando a semente de cártamo, observaram aumento na porcentagem de novilhas na puberdade e na concentração de progesterona, mas não ocorreu para a concentração de insulina.

Lammoglia et al. (2000), sugerem que a resposta à suplementação de gordura pode ser raça-dependente, pois novilhas com baixa gordura corporal podem ter uma exigência de gordura diferente das novilhas com maiores composições de gordura e, podem se beneficiar do suplemento.

As novilhas suplementadas com semente de linhaça apresentaram maior $(p<0,05)$ número de cistos ovário direito $(4,6)$ em relação às novilhas sem gordura na ração $(1,4)$ ou àquelas suplementadas com semente de canola $(1,3$, tabela VI). Não houve diferença $(p>0,05)$ entre estes dois últimos tratamentos. No entanto, o número de cistos observados no ovário esquerdo foi semelhante entre os três tratamentos. Em razão do maior número de cistos presentes no ovário direito das novilhas suplementadas com semente de linhaça, o somatório do número de cistos dos ovários direito + esquerdo, foi maior $(p<0,05)$ para as novilhas suplementadas com semente de linho $(8,6)$, em relação às novilhas suplementadas com semente de canola $(3,7)$ ou das novilhas não suplementadas $(4,20)$, sendo que não houve diferença $(\mathrm{p}>0,05)$ entre os dois últimos tratamentos (tabela VI).

As novilhas suplementadas com semente de linhaça apresentaram o menor $(\mathrm{p}<0,05)$ número de estruturas totais $(5,33)$

Archivos de zootecnia vol. 58, núm. 222, p. 248. 
em relação às novilhas dos tratamentos sem gordura $(10,40)$ e suplementadas com semente de canola $(8,67)$, sendo que não houve diferença $(p>0,05)$ entre os dois últimos tratamentos (tabela VII).

$\mathrm{O}$ menor número de estruturas totais observados nos animais tratados com linhaça pode estar relacionado ao maior número de cistos encontrados para este tratamento, pois o cisto é um folículo dominante que falha em ovular (Butler, 2000).

Rigolon(2001) observou 2,36; 3,29 e 2,00 para novilhas $1 / 2$ Nelore $x \quad 1 / 2$ Simental, recebendo níveis de ingestão de matéria seca (IMS) de 1,2; 1,6 e 2,6\% de IMS, respectivamente. Da mesma forma, Santiago et al. (2001), observaram valores de número de estruturas totais de 4,7 para novilhas Nelores superovuladas. Os dados obtidos com novilhas no presente experimento, para os tratamentos SGO e CAN, são semelhantes aos obtidos por vacas Nelores, coletados por Pinto Neto et al. (2000), com número de estruturas totais de 10,8 . O número de estruturas totais observados neste experimento evidencia a boa resposta dos SGO e

Tabela VII. Fontes de gordura sobre o número de estruturas totais, estruturas transferíveis, estruturas degeneradas, estruturas não fertilizadas e viabilidade das estruturas. (Fat sources on number of and total, transferable and degenerate structures, non-fertilized structure and viability of structures)

\begin{tabular}{|c|c|c|c|}
\hline Item & SGO & LIN & CAN \\
\hline$N^{\circ}$ novilhas & 6 & 6 & 5 \\
\hline $\mathrm{N}^{\circ}$ novilhas $\mathrm{CL}$ & 5 & 6 & 3 \\
\hline $\mathrm{N}^{0}$ Estruturas totais & $10,4^{a}$ & $5,3^{b}$ & $8,7^{\mathrm{a}}$ \\
\hline $\mathrm{N}^{\circ}$ Estruturas transferíveis & $2,8^{b}$ & $1,2^{c}$ & $6,7^{\mathrm{a}}$ \\
\hline $\mathrm{N}^{\circ}$ Estruturas degeneradas & $7,6^{b}$ & $3,7^{\mathrm{a}}$ & $2,0^{\mathrm{a}}$ \\
\hline$N^{\circ}$ Estruturas não fertilizadas & $0,2^{\mathrm{b}}$ & $0,5^{b}$ & $0,0^{\mathrm{a}}$ \\
\hline Viabilidade das estruturas & $26,9^{b}$ & $22,0^{\mathrm{b}}$ & $76,9^{\mathrm{a}}$ \\
\hline
\end{tabular}

${ }^{1}$ Sem de gordura; ${ }^{2}$ Semente de linho; ${ }^{3}$ Semente de canola.

ab $\mathrm{Na}$ mesma linea, médias com letras diferentes apresentaram diferença estatística $(p<0,05)$.
CAN em novilhas de corte, pois estes foram semelhantes a vacas, que já atingiram a maturidade sexual completa.

$\mathrm{O}$ menor $(\mathrm{p}<0,05)$ número de estruturas transferíveis $(1,17)$ foi obtido nos animais tratados com semente de linhaça e maior com aqueles tratados com canola. Embora, as novilhas suplementadas com semente de linho tivessem apresentado $100 \%$ de resposta superovulatória e sendo fonte de ômega-3, características consideradas benéficas por Petit et al. (1998), estas não refletiram sobre a qualidade dos embriões coletados.

Ao contrário, as novilhas suplementadas com semente de canola que resultaram no efeito responsivo negativo, apresentaram o maior $(\mathrm{p}<0,05)$ número de estruturas transferíveis $(6,67)$. Logo, as concentrações de ácido erúcico e glicosinolatos presentes na semente de canola não são embrio-tóxicas, evidenciadas também pelo menor número de estruturas degeneradas. As novilhas que não receberam gordura na ração apresentaram um número intermediário $(2,80)$ de estruturas transferíveis, consideradas dentro do normal para novilhas Nelore.

Os resultados obtidos para o número de embriões transferíveis para animais tratados com semente de canola, foram superiores aos encontrados por Santiago et al. (2001) de 2,4, superovulando 17 novilhas Nelore, com FSH. Pinto Neto et al. (2000) verificaram 6,18 embriões transferíveis ao superovularem 30 vacas Nelore com 350 UI de FSH (Pluset ${ }^{\circledR}$ ).

Por outro lado, o maior $(\mathrm{p}<0,05)$ número de estruturas degeneradas foi observado nas novilhas sem suplementação de gordu$\mathrm{ra}(7,60)$ em relação às novilhas suplementadas com semente de linho $(3,67)$ e semente de canola $(2,00)$. Não houve diferença ( $\mathrm{p}>0,05)$ no número de estruturas degeneradas entre as novilhas dos tratamentos com semente de linho ou canola. Segundo O'Callanghan e Bolanda (1999), qualquer alteração existente na concentração de progesterona no período inicial do embrião 


\section{MULLER ET AL.}

pode resultar na morte e/ou degeneração do mesmo. Além disso, a progesterona é de suma importância para a regulação do funcionamento do sistema reprodutor feminino, sendo esta principalmente sintetizada pelo corpo lúteo, oriundo da reorganização celular dos folículos após a ovulação (Borges et al., 2001). Logo, estes resultados podem ser explicados pela hipótese vinculada à síntese de progesterona, pois o fornecimento de gordura aumenta as concentrações de colesterol sangüíneo (Lammoglia et al., 1997) e este possui relação de causa e efeito sobre a síntese de progesterona, por ser precursor da mesma (Lammoglia et al., 2000; Oldick et al., 1997).

As novilhas suplementadas com semente de canola não apresentaram estrutura não fertilizada. Por outro lado, o número de estruturas não fertilizadas foi maior $(\mathrm{p}<0,05)$ nas novilhas suplementadas com sementes de linhaça $(0,50)$ ou sem suplementação de gordura $(0,20)$. O baixo número de estruturas não fertilizadas encontrado neste experimento, quando comparados àquelas de Rigolon (2001), Pinto Neto et al. (2000) e Santiago et al. (2002), podem ser explicados em função de ter sido utilizado sêmen fresco, para a inseminação artificial das novilhas, com excelente qualidade, por apresentar $70 \%$ de motilidade e vigor 4. Além disso, possivelmente as novilhas suplementadas com semente de canola não apresentaram ovulações tardias, decorrente do tipo de ácido graxo presente (ômega-9).

Em vista do maior $(\mathrm{p}<0,05)$ número de estruturas transferíveis e do menor $(\mathrm{p}<0,05)$ número de estruturas degeneradas, as

\section{BIBLIOGRAFIA}

Bett, V., G.T. Santos, L.J.M. Aroeira, P.G. Dias, K.P. Faria, L.M. Zeoula e T.C.S.S. Leggi. 1999. Digestibilidade in vivo de cordeiros alimentados com canola em grão integral em diferentes formas. Em: Reunião Anual da Sociedade Brasileira de Zootecnia, 36, 1999. Porto Alegre. Anais... SBZ. Porto Alegre. novilhas suplementadas com semente de canola apresentaram a maior viabilidade $(p<0,05)$ das estruturas $(76,93 \%)$. Este valor foi superior ao encontrado por Pinto Neto et al. (2000) de 57,46\%, com vacas Nelores e Rigolon (2001), de 50,0; 65,4 e 48,0\% para novilhas $1 / 2$ Nelore $x 1 / 2$ Simental, recebendo níveis de ingestão de matéria seca de 1,2; 1,6 e 2,6\% em relação ao peso vivo.

As novilhas suplementadas com semente de linhaça $(21,95 \%)$ e sem gordura $(26,92 \%)$ apresentaram semelhantes viabilidades, mostrando que a fonte de ômega- 9 causou efeitos benéficos na qualidade dos embriões coletados. No entanto, os mecanismos que interferem na viabilidade dos embriões ainda são complexos.

\section{CONCLUSÕES}

A inclusão de diferentes fontes de ácidos graxos (ômega-3, semente de linho ou ômega-9, semente de canola) na ração não alterou o desempenho de novilhas Nelores terminadas em confinamento e alimentadas ad libitum, em relação àquelas alimentadas sem a inclusão de gordura.

Indica-se a inclusão de semente de canola (fonte de ácidos graxos, ômega-9), na dieta de fêmeas a serem superovuladas em função da melhor produção de embriões.

\section{AGRADECIMENTOS}

Ao Conselho Nacional de Desenvolvimento Científico e Tecnológico (CNPq) pela concessão das bolsas de Pesquisador 1B (Ivanor Nunes do Prado) e Pós doutorado Júnior (Robério Rodrigues Silva).

Borges, A.M., A.A. Torres e J.R.M. Ruas. 2001 Concentrações plasmáticas de progesterona e metabólitos lipídicos em novilhas mestiças tratadas ou não com hormônio do crescimento e superovuladas. Rev. Bras. Zootecn., 30: 16891696.

Butler, W.R. 2000. Nutritional interactions with

Archivos de zootecnia vol. 58, núm. 222, p. 250. 


\section{FONTES DE GORDURA SOBRE DESEMPENHO E PRODUÇÃO DE EMBRIÕES}

reproductive performance in dairy cattle. Anim. Reprod. Sci., 60-61: 449-457.

Del Vecchio, R.P., C.C. Chase Jr., P. Bastidas and R.D. Randel. 1990. Oxytocin-induced changes in plasma 13,14-dihydro-15-keto-prostglandin $F_{2 \alpha}$ concentrations on days 10,20 and 30 postpartum in the bovine. J. Anim. Sci., 68: 4261-4266.

Downey, R.K. 1990. Canola: a quality brassica oilseed. Timber Press. Portland, Or. USA. $215 \mathrm{p}$.

Gomez, K.A. and A.A. Gomez. 1984. Statistical procedures for agricultural research. $2^{\text {nd }}$ ed. John Wiley. New York. 680 p.

IETS. 1999. Manual da Sociedade Internacional de Transferência de Embriões. Trad. Enoch Borges de Oliveira Filho. $3^{\text {a }}$ ed. SBTE. São Paulo. p. 180

Lammoglia, M.A., R.A. Bellows, E.E. Grings, J.W. Bergman, R.E. Short and M.D. MacNeil. 2000. Effects of dietary fat and sire breed on puberty, weight, and reproductive traits of $\mathrm{F} 1$ beef heifers. J. Anim. Sci., 78: 2244-2252.

Lammoglia, M.A., S.T. Willard and D.M. Hallford. 1997. Effects of daietary fat follicular development and circulating concentration of lipids, insulin, progesterone, estradiol $17 \mathrm{~b}$, 13,14-dihydro-15-keto-prostraglandin $f_{2} \alpha$ and growth hormone in estrus cyclic Brahman cows. J. Anim. Sci., 75: 1591-1600.

Marques, J.A., I.N. Prado, W.G. Nascimento, L.P. Rigolon e M.L.D. Sakuno. 2001. Avaliação do desempenho de novilhas mestiças em diferentes estádios reprodutivos. Em: Reunião Anual da Sociedade Brasileira de Zootecnia, 38, 2001. Piracicaba. Anais... SBZ. Piracicaba.

Medroni, S., I.N. Prado e W.G. Nascimento. 2000. Efeito da combinação de dietas contendo milho ou triticale e farelo de soja ou levedura sobre o desempenho de novilhas nelore terminadas em confinamento. Acta Scient., 22: 787-791.

Müller, L. 1987. Normas para a avaliação de carcaças e concurso de carcaça de novilhos. Imprensa Universitária. Santa Maria. $31 \mathrm{p}$.

NRC. 1996. Nutrient requirements of beef cattle. $7^{\text {th }}$ Rev. Ed. National Academy Press. Washington, D.C. $242 \mathrm{p}$.

O'Callanghan, D. and M.P. Bolanda. 1999. Nutritional effects on ovulation, embryo development and the establishment of pregnancy in ruminants. Anim. Sci., 68: 299-314.

Ohashi, O.M., S.H.F. Costa e A.C.M. Machado.
1998. Características ovarianas de vacas zebuínas (Bos indicus) e bubalinas (Bubalus bubalis). Arq. Faculdade de Veterinária UFRGS, 26: 311.

Oldick, B.S., C.R. Staples, W.W. Thatcher and P. Gyawu. 1997. Abomasal infusion of glucose and fat-effect on digestion, production, and ovarian and uterine functions of cows. J. Dairy Sci., 80: 1315-1328.

Petit, H.V., J.G. Dewhurst and J.G. Proulx. 1998. Milk yield and reproduction of dairy cows fed satured or unsatured fat. J. Dairy Sci., 81 (Suppl. 1): 302

Petit, H.V., R.J. Dewhurst and N.D. Scollan. 2002. Milk production and composition, ovarian function, and prostaglandin secretion of dairy cows fed omega-3 fats. J. Dairy Sci., 85: 889899.

Petit, H.V., R. Rioux, P.S. D'oliveira and I.N. Prado. 1997. Performance of growing lambs fed grass silage with raw or extrused soybean or canola seeds. Can. J. Anim. Sci., 77: 455-463.

Pinto Neto, A., J.M. Silva Filho e J.F. Fonseca. 2000. Desempenho de doadoras da raça Nelore em programa de transferência de embriões. Arq. Faculdade de Veterinária UFRGS, 27: 311.

Prado, I.N., A.S. Martins, C.R. Alcalde, L.M. Zeoula e J.A. Marques. 2000. Desempenho de novilhas alimentadas com rações contendo milho ou casca de mandioca como fonte energética e farelo de algodão ou levedura como fonte protéica. Rev. Soc. Bras. Zootecn., 29: 278287.

Rigolon, L.P. 2001. Ingestão de diferentes níveis de matéria seca e condição corporal sobre o desempenho zootécnico, digestibilidade aparente, alterações metabólicas, perfil hormonal e resposta reprodutiva á superovulação, em fêmeas bovinas de corte. Tese (Doutorado em Zootecnia). Universidade Estadual de Maringá. Maringá. $113 p$.

Romans, J.R., R.C. Johnson, D.M. Wulf, G.W. Libal and W.J. Costello. 1995. Effects of ground flaxseed in swine diets on pig performance and on physical and sensory characteristics and omega-3 fatty acid content of pork: I. Dietary level of flaxseed. J. Anim. Sci., 73: 1982-1986.

Santiago, L.L., C.A.A. Torres e E.P. Costa. 2001. Influência do folículo dominantes na resposta superovulatória de novilhas de corte. Em: 


\section{MULLER ET AL.}

Reunião Anual da Sociedade Brasileira de Zootecnia, 38, 2001. Piracicaba. Anais... SBZ. Piracicaba.

Santiago, L.L., C.A.A. Torres e E.T. Nogueira. 2002. Folículo dominante e resposta superovulatória em novilhas da raça Nelore. Rev. Bras. Zootecn., 31 (Supl.): 363-368.

Sas Institute. 1996. SAS/STAT. User's guide. $11^{\text {a }}$ ed. SAS Inst. Cary, NC.

Staigmiller, R.B., R.A. Bellows and R.E. Short. 1993. Conception rates in beef heifers following embryo transfer at the pubertal or third estrus. Theriogenology, 39: 315 (Abstract).

Staples, C.R., W.W. Thatcher and J.M. Burke. 1996. Influence of supplemental fat on reproductive tissues of the dairy cow. J. Dairy Sci., 79: 1964-1978.

Thomas, M.G. and G.L. Williams. 1996. Metabolic hormone secretion and FSH-induced superovulatory responses of beef heifers fed dietary fat supplements containing predominantly saturated or polyunsaturated fatty acids. Theriogenology, 45: 451-458.

Thomas, M.G., B. Bao and G.L. Williams. 1997. Dietary fats varying in their fatty acid composition differentially influence growth in cows fed isoenergetic diets. J. Anim. Sci., 75: 25122519.

Zinn, R.A. 1989. Influence of level and source of dietary fat on its comparative feeding value in finishing diets for steers: feedlot cattle growth and performance. J. Anim. Sci., 67: 1029-1037.

Zinn, R.A. and A. Plascencia. 1996. Effects of forage level on the comparative feeding value of supplemental fat in growing-finishing diets for feedlot cattle. J. Anim. Sci., 74: 1194-1201.

Archivos de zootecnia vol. 58, núm. 222, p. 252. 